\title{
An Air Bearing Facility for Antenna Pointing Mechanism
}

\author{
Guoyong Yang ${ }^{1,2,}{ }^{*}$, Hongguang Wang ${ }^{1}$, Lie Ling $^{1}$ and Feng Gao ${ }^{3}$ \\ ${ }^{1}$ Shenyang Institute of Automation Chinese Academy of Sciences, Shenyang 110016, China \\ ${ }^{2}$ University of Chinese Academy of Sciences, Beijing 100049, China \\ ${ }^{3}$ Beijing Institute of Control Engineering, Beijing 100080, China \\ *Corresponding author
}

\begin{abstract}
An air bearing facility is built to test the antenna pointing mechanism of track and data relay satellite. A hierarchical and simultaneous gravity unloading method is proposed to unload the gravity on the ground. The air bearing facility is a two layers structure. Planar air bearings and air spindle are used to unload the gravity of antenna pointing mechanism. An artificial load is designed to replace the antenna. Two joints of antenna pointing mechanism can rotate separately or together freely without the influence of gravity on the ground. The artificial load not only matches the mass, moment of inertial and basic frequency of the antenna, but also solves the coupling problem of moment of both axes. The air bearing facility is designed and established based on the proposed method and analysis. The effectiveness of this facility is proved with the simulation and the experiments and test results.
\end{abstract}

Keywords — air bearing facility; planar air bearing; air spindle; unload gravity; antenna pointing mechanism

\section{INTRODUCTION}

Air bearing simulator is designed to simulate the micro-gravity environment on the ground. It is used to test the performance of aircraft [1, 2]. Traditional air bearing simulators can unload the gravity of whole aircraft such as a satellite. The control, rotation or formation flight performance can be tested on the simulator [3,4]. Traditional simulator can also unload the gravity of each link of robot arm. MIT has built several 3-DOF (degree of freedom) air bearing simulators to test the control method of small satellites formation flight [5-7]. Those simulators can move on the horizontal plane and rotate around the vertical axis. German company EDAS Astrium also develops 5-DOF air bearing simulator to verify software and software-hardware test for multi-purpose satellites [8]. Beijing Institute of Control Engineering designs a single-axis kinematic simulator for antenna pointing mechanism [9, 10]. Satellite technology institute of Harbin Institute of Technology develops a 5-DOF air bearing simulator $[2,11]$. Stanford Aerospace Lab has built an air bearing simulator for a two-link arm [12, 13], which can only unload gravity while floating on horizontal plane. This simulator uses a planar air bearing to unload the gravity on the end of the arm. Because of the mechanical structure, all the above simulators can either unload the gravity of whole aircraft or unload the gravity of parallel joints.
The objects can only float on the horizontal plane or rotate around vertical joint.

Tracking and data relay satellites (TSRD) can provide data relaying, continuous tracking and orbit monitoring services [14] between spacecraft (in low and medium orbit), spacecraft and ground stations [14]. Two large antennas are mounted on the TDRS to capture target vehicle [15]. The antenna is driven by the antenna pointing mechanism (APM), so the performance of the APM is a key part of the performance of the whole satellite. The APM consists of two joints which are orthogonal and traditional simulator cannot unload its gravity, then a new facility is needed to test the performance of the APM while the load is mounted [16].

\section{HIERARCHICAL AND SimUltanEOUS GRAVITY UNLOADING METHOD}

The APM consists of two orthogonal joints. The APM can be divided into three parts: vertical joint stator, vertical joint rotor and horizontal joint stator, horizontal joint rotor. Vertical joint stator is mounted on the satellite while the antenna is mounted on the horizontal joint rotor. Vertical joint rotor and horizontal joint stator is one piece. The goal is to unload the influence of gravity on two joint.

The facility should offer two rotational DOF. Planar air bearing, spherical air bearing and air spindle are used to unload gravity. Planar air bearing and air spindle are used to build the facility (shown in Figure I) based on the analysis of APM structure and characteristics of three kinds of air bearings. Planar air bearing is used to unload the gravity of vertical joint, while air spindle is used to unload the gravity of horizontal joint.

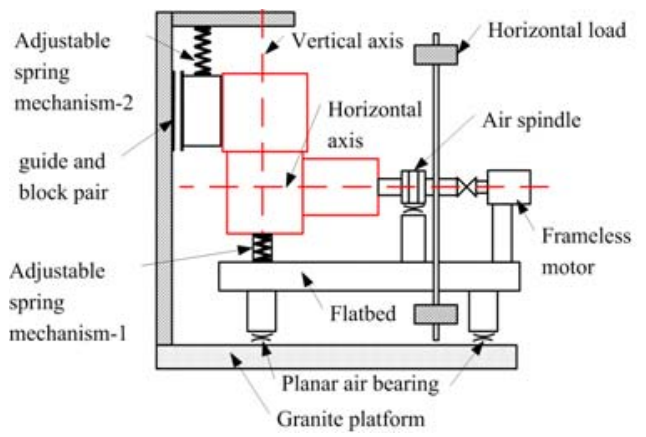


(A)

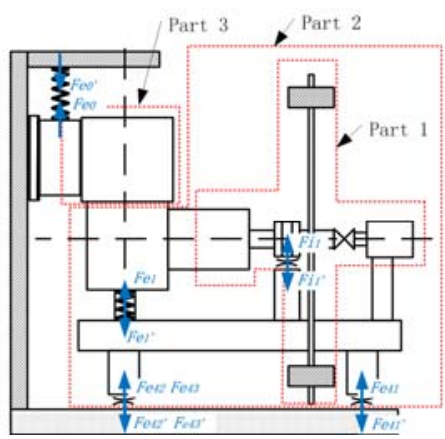

(B)

\section{FIGURE I. SCHEME OF GRAVITY UNLOADING METHOD}

Hierarchical and simultaneous gravity unloading method means that the air bearing facility is a two-layer structure (air spindle is used to unload the gravity of horizontal joint on the upper layer, planar air bearing is used to unload the gravity of vertical joint on the lower layer) and unload the gravity while both axes rotate one after another or together. This configuration uncouples two joints during the gravity unloading, thus the APM can rotate two joints freely without the influence of gravity. As shown in the figure, the main frame is mounted on the granite platform, vertical joint stator of APM is mounted on the main frame by the guide and block pair. Adjustable spring mechanism-2 is used to hold the position of APM vertical joint stator. Artificial load (to replace the antenna) is fixed onto the horizontal joint rotor through the air spindle rotor. Air spindle stator is fixed on the support flatbed. A frameless motor is equipped to simulate the influence of signal cable of antenna. Rotor of frameless motor is fixed coaxially with the rotor of air spindle. Stator of frameless motor and stator of air spindle are mounted on the support flatbed. Adjustable spring mechanism-1 is mounted between the support flatbed and vertical joint rotor of APM. Three planar air bearings are mounted under the support flatbed. Those air bearings can float on the granite platform.

Figure I (B) shows the hierarchical and simultaneous gravity unloading method. Firstly, the air spindle unloads the gravity of part 1 . Horizontal joint rotor of APM, air spindle rotor, horizontal load and frameless motor rotor are fixed coaxially. The gravity center of part 1 coincides with the center of air spindle. The gap between the rotor and stator of air spindle is full of high-pressure air when the facility is working. So there is only viscous resistance while the horizontal joint of APM is rotating, just as rotates in space. Secondly, the gravity of vertical joint rotor of APM is unloaded by the adjustable spring mechanism- 1 and planar air bearings. The adjustable spring mechanism-1 unloads the gravity of vertical joint rotor of APM precisely by adjusting the force of spring. The adjustable spring mechanism-1 can be fixed on the support flatbed stably because the contact area between the adjustable spring mechanism- 1 and vertical joint rotor is a square plane. Three planar air bearings are placed as a triangle to support part 2 and offer the rotational DOF for vertical joint. There will be establish and disappear process during the initiate and power off of the air bearing facility, so the APM may move vertically in a small range during those two steps. Guide and block pair and adjustable spring mechanism-2 are applied to free the vertical movement and hold the APM at the same time.

\section{HORIZONTAL LOAD DESIGN}

A payload is necessary to test the performance of APM, but the actual antenna is unavailable. An artificial payload is designed to simulate the moment of inertia and frequency. The constraints are as follow taken the capacity of the granite platform, rotation angle of two joint and the mass of artificial payload into consideration:

$$
\begin{aligned}
& \left\{\begin{array}{l}
J_{\text {min }} \leq J_{h} \leq J_{\max } \\
J_{\text {min }} \leq J_{v} \leq J_{\text {max }}
\end{array}\right. \\
& \text { freq }_{\min } \leq \text { freq } \leq \text { freq }_{\max } \\
& \text { s.t. } \theta_{h} \geq \theta_{1} \\
& \theta_{v} \geq \theta_{2} \\
& \sum_{H} m_{i} \leq m_{\operatorname{maxH}} \\
& \sum_{V} m_{i} \leq m_{\operatorname{maxV}}
\end{aligned}
$$

Where, $\sum_{H} m_{i}$ and $\sum_{V} m_{i}$ are payload mass of horizontal and vertical joint respectively. ${ }^{\theta_{h}}$ and $\theta_{v}$ are angle range of horizontal and vertical joint. $J_{h}$ and $J_{v}$ are moment of inertia of horizontal and vertical joint respectively. Moment of inertia is the function of mass distribution, $J_{h}=\int_{h} \Delta m_{i} r_{i}^{2}, J_{v}=\int_{v} \Delta m_{i} r_{i}^{2}$. freq is the basic model of horizontal load. Hierarchical analysis method is applied to design the artificial load because there is not a structure with parameters to be optimized.

The configuration of horizontal load is analyzed firstly. The load should be either dumbbell or triangular star with the constraint of rotational angle range, as shown in the figure.

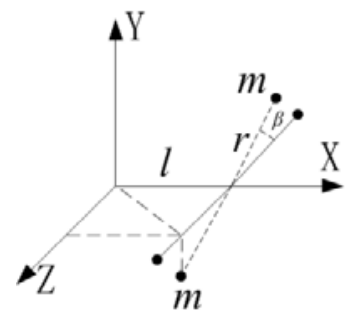

(A)

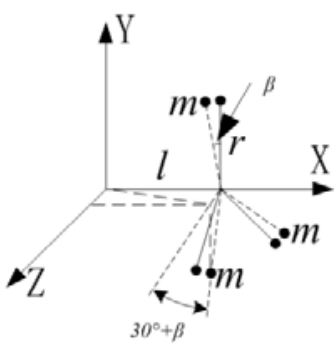

(B)

\section{FIGURE II. STRUCTURE OF HORIZONTAL LOAD}

In Figure II (A), the moment of inertia around vertical axis is: 


$$
J_{y a}=2 \times m\left(\sqrt{(r \cos \beta)^{2}+l^{2}}\right)^{2}=2 m l^{2}+2 m r^{2} \cos ^{2} \beta
$$

The moment of inertia changes with the angle of horizontal joint. In Figure II (B), the moment of inertia around vertical axis is:

$$
J_{j b}=m\left(\sqrt{(r \cdot \sin \beta)^{2}+l^{2}}\right)^{2}+m\left(\sqrt{\left(r \cdot \cos \left(30^{\circ}+\beta\right)\right)^{2}+l^{2}}\right)^{2}+m\left(\sqrt{\left(r \cdot \cos \left(30^{\circ}-\beta\right)\right)^{2}+l^{2}}\right)^{2}=3 m l^{2}+\frac{3}{2} m r^{2}
$$

The moment of inertia remains constant while horizontal joint is rotating. So the horizontal load is designed with the triangular star configuration which uncouples the moment of inertia of two joint.

Then the horizontal load should satisfy the requirement of frequency. The free vibration differential equation of n-DOF structure is:

$$
[M]\{X\}+[K]\{X\}=\{0\}
$$

Where, $[\mathrm{M}]$ is the $\mathrm{n}$ rank mass matrix of structure. $[\mathrm{K}]$ is the $\mathrm{n}$ rank stiffness matrix. $\{X\}$ and $\{X\}$ are the acceleration and displacement in the reference coordinate. The following equation is got after derivation:

$$
\operatorname{det}\left(-\omega^{2}[M]+[K]\right)=0
$$

Natural frequencies are calculated: $\omega 1, \omega 2$, , $\omega n$. Each frequency is a function of stiffness matrix and mass matrix. So the mass and stiffness are adjusted after the initial design and frequencies calculation, then the frequencies are calculated again. Repeat this process until the basic frequency of final structure satisfies the requirement. The deformation and stress concentration are reduced after final optimization. The final design is shown in the following:

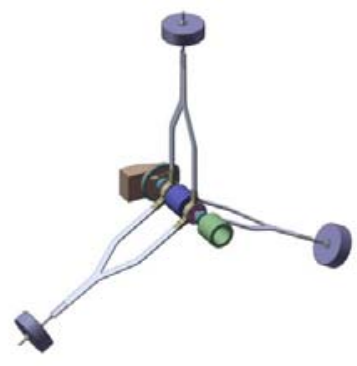

(A) STRUCTURE 1

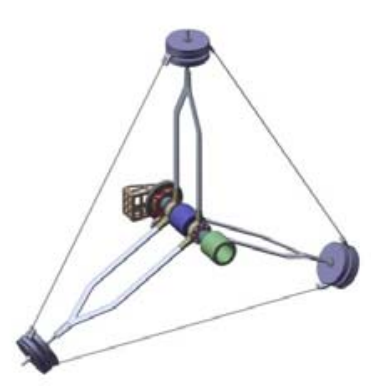

(B) STRUCTURE 2

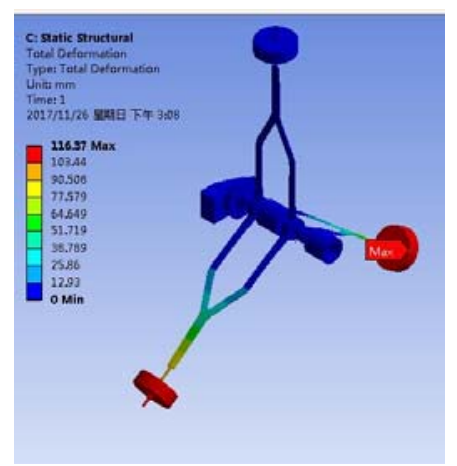

(C) DEFORMATION OF STRUCTURE 1

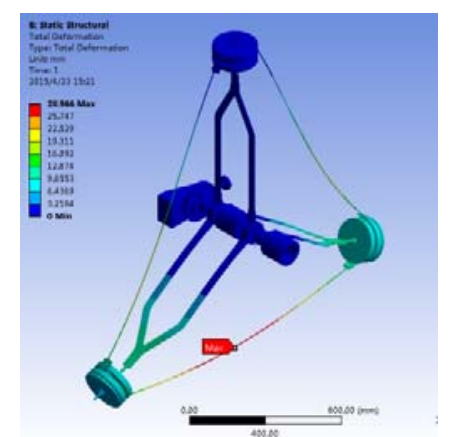

(D) DEFORMATION OF STRUCTURE 2

FIGURE III. HORIZONTAL LOAD

\section{AIR BEARING FACILITY TEST}

The air bearing facility is designed and established based on the analysis, shown in Figure IV. 


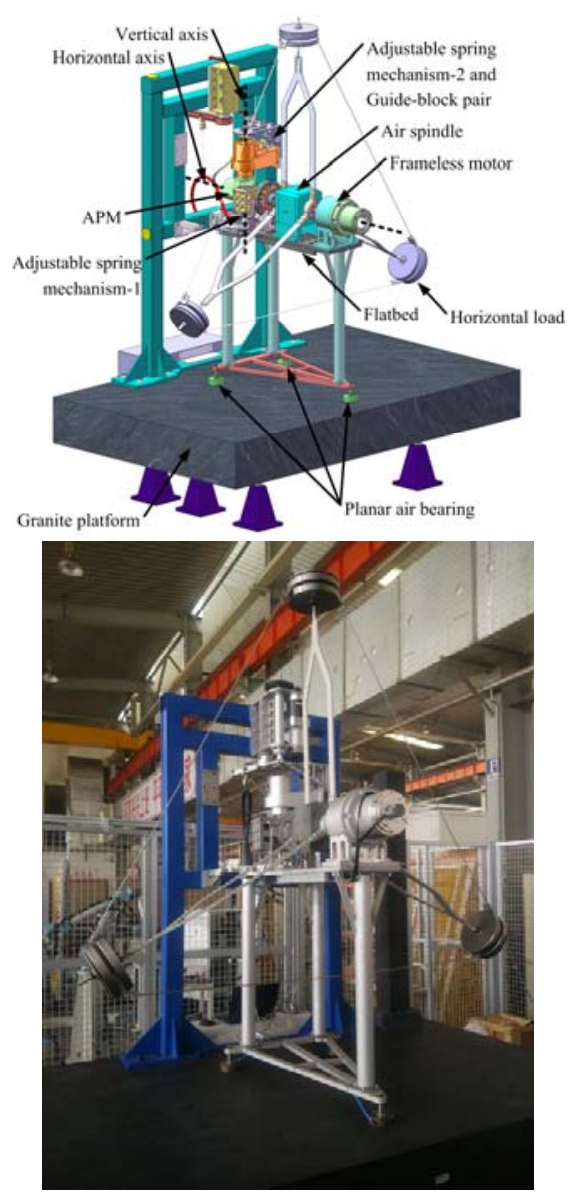

FIGURE IV. AIR BEARING FACILITY

Gravity unloading precision is tested on the facility. The precision is calculated with the output of torque sensor on the facility and theory calculation. After the initiate of the facility, both joint rotate with the process of Static-Acceleration-Uniform-Deceleration-Static. Acceleration is $\pm 0.1 \% \mathrm{~s} 2$

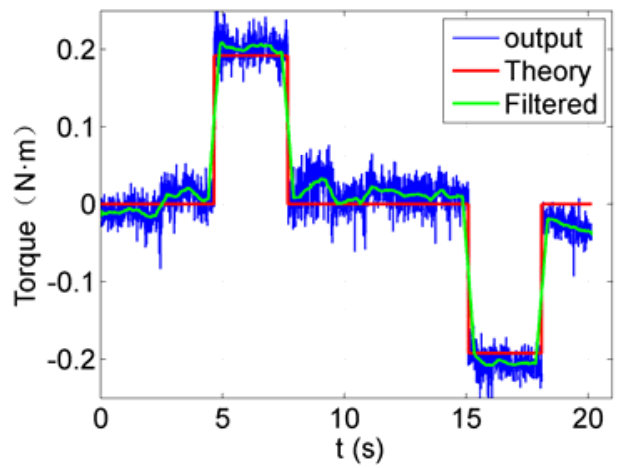

(A) TORQUE OF VERTICAL JOINT

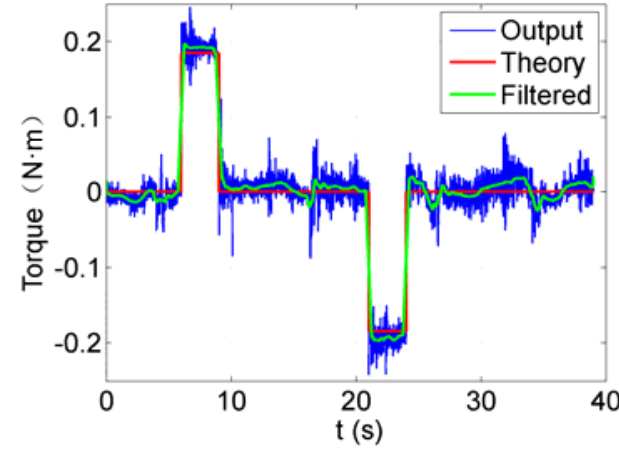

(B) TORQUE OF HORIZONTAL JOINT

FIGURE V. TORQUE OF SENSOR AND THEORY CALCULATION

There are deviations between sensor output curve and theory calculation especially during the acceleration and deceleration. Compare the filtered curve and theory curve, the precision of gravity unloading is $90 \%$. The precision reduce is caused by the tolerance of manufacture, assembly, viscous resistance, etc. which are inevitable.

\section{SUMMARY}

A hierarchical and simultaneous gravity unloading method is proposed based on the analysis of antenna pointing mechanism. This method can unload the gravity of two orthogonal joint with air spindle and planar air bearings. The artificial load is designed and satisfies the constraints of moment of inertia and basic frequency. The artificial load also uncouples the moment of two joint. An air bearing facility is designed and established based on the method and analysis of load. The test results show that the precision of gravity unloading is $90 \%$.

\section{ACKNOWLEDGEMENT}

This research was supported by the State Key Laboratory of Robotics.

\section{REFERENCES}

[1] XU Jian, YANG Qingjun, BAO Gang, et al. Research on lateral Disturbance Force of the 5-DOF Air-bearing Spacecraft Simulator. Journal of Astronautics, 2009. 30(5):p.1823-1828

[2] XU Jian. Prototype Development and Research on Key Thchnology of the Five Degrees of Freedom Air bearing Spacecraft Simulator. Harbin Insitute of Technology.

[3] LI Jisu, Mu Xiaogang, ZHANG Jinjiang. Physical Simulation for Satellite Control System. Aerospace Contorl, 2004. 22(2): p. 37-41, 45.

[4] Schwartz, J.L., M.A. PeckC.D. Hall, Historical review of air-bearing spacecraft simulators. Journal of Guidance, Control, and Dynamics, 2003. 26(4): p. 513-522.

[5] Chung, S.-J., Nonlinear control and synchronization of multiple Lagrangian systems with application to tethered formation flight spacecraft. 2007, Massachusetts Institute of Technology.

[6] Chung, S.-J., et al. Multidisciplinary control of a sparse interferometric array satellite test-bed. in Proc. AIAA Guidance, Navigation and Control Conf. 2003.

[7] Saenz-Otero,A.,et al. SPHERES: Development of an ISS laboratory for formation flight and docking research. in IEEE Aerospace Conference. 2002. 
[8] ZHANG Shijie, CAO Xibin. Physical Simulation System for Spacecraft Rendezvous and Docking Based on MicroSim. Aerospace Control, 2006(02): p. 63-67+96.

[9] LI Jisu, ZENG Haibo, LI Tieshou, et al. Ground Physical Simulation Experiment System for Combined Control of Multibody Satellite. Control Engineering, 2002(06): p. 14-18.

[10] LI Jisu, ZENG Haibo, LI Tieshou. Physical Simulation Test System for Combined Control of Multibody Satellite. Aerospace Contorl, 2003. 21(2): p. 27-32.

[11] XU Jian, YANG Qingjun, BAO Gang, et al. Research and Development of Air Bearing Spacecraft Simulator on the Multiple Degree o fFreedom. Aerospace Control, 2009. 27(6): p. 96-101.

[12] Schubert, H.C.J.P. How, Space construction: an experimental testbed to develop enabling technologies, in Telemanipulator and Telepresence Technologies Iv, M.R. Stein, Editor. 1997, Spie-Int Soc Optical Engineering: Bellingham. p. 179-188.

[13] Aeronautics, S.U.D.o., et al., Manipulation of a Free-floating Object Using a Macro/mimi-manipulator with Structural Flexibility. 1997.

[14] LI Weidong, HOU Lihong. Reaearch on High-speed Train Position Technology Based on Satellite Navigation System. Information and Control, 2016(04): p. 479-486.

[15] ZHAO Yanzhi, ZHAO Fei, YANG Jiantao, et al. Modeling and Analysis of 6-RUS Parallel Mechanism of Image Compensation of Satellite Camera. China Mechanical Engineering, 2016(03): p. 369-375.

[16] CHEN Yuhang, ZHAO Tieshi, SONG Xiaoxin, et al. Analysis of DOF Properties and Kinematics for Variable Configuration Parallel Multi-dimensional Vibration Platform. ROBOT, 2016(02): p. 135-143. 